\title{
Theory-Driven Design Strategies for Technologies that Support Behavior Change in Everyday Life
}

\author{
Sunny Consolvo ${ }^{1,2}$, David W. McDonald ${ }^{2}$, \& James A. Landay ${ }^{1,3}$ \\ ${ }^{1}$ Intel Research Seattle \\ Seattle, WA 98105 USA \\ sunny.consolvo@intel.com \\ ${ }^{2}$ The Information School
DUB Group
University of Washington
Seattle, WA 98195 USA
dwmc@u.washington.edu \\ ${ }^{3}$ Computer Science \& Engineering \\ DUB Group \\ University of Washington \\ Seattle, WA 98195 USA \\ landay@cs.washington.edu
}

\begin{abstract}
In this paper, we propose design strategies for persuasive technologies that help people who want to change their everyday behaviors. Our strategies use theory and prior work to substantially extend a set of existing design goals. Our extensions specifically account for social characteristics and other tactics that should be supported by persuasive technologies that target long-term discretionary use throughout everyday life. We used these strategies to design and build a system that encourages people to lead a physically active lifestyle. Results from two field studies of the system-a three-week trial and a three-month experiment-have shown that the system was successful at helping people maintain a more physically active lifestyle and validate the usefulness of the strategies.
\end{abstract}

\section{Author Keywords}

Design strategies, behavior change, persuasive technology, physical activity, mobile phone, everyday life, lifestyle.

\section{ACM Classification Keywords}

H.5.2. User Interfaces, H.5.m. Miscellaneous.

\section{INTRODUCTION}

A recent trend in computing is to develop persuasive technologies [7] to help people change their everyday behaviors in support of the lifestyle they wish to lead $[3,8,10,11,13,14,15,18]$. For this paper, we define a lifestyle as a pattern of behaviors that an individual enacts and that characterizes who she is and how she is perceived. Thus, a lifestyle results from the choices that an individual makes, and she may make different choices to change that lifestyle.

However, getting an individual to change her everyday behaviors, and hence her lifestyle, is challenging. People want to be financially secure, yet consumer debt is on the rise. People want to be fit and healthy, yet physical inactivity and poor eating habits are leading to serious health problems. The discrepancy between desired and

Permission to make digital or hard copies of all or part of this work for personal or classroom use is granted without fee provided that copies are not made or distributed for profit or commercial advantage and that copies bear this notice and the full citation on the first page. To copy otherwise, or republish, to post on servers or to redistribute to lists, requires prior specific permission and/or a fee.

CHI 2009, April 4-9, 2009, Boston, MA, USA.

Copyright 2009ACM 978-1-60558-246-7/08/04...\$5.00 actual lifestyle can be in part attributed to simple everyday decisions. Sometimes those decisions support her desired lifestyle; other times they do not. Often, it is a pattern of "poor" decisions that prevents the individual from achieving her desired lifestyle [17]. Making an occasional poor or arbitrary decision is seldom a serious problem.

Fortunately, behavior and how behavior is influenced is well studied in the field of psychology. Designers of persuasive technologies often draw from behavioral theories such as Goal-Setting Theory [12] or the Transtheoretical Model of Behavior Change [16]. While such traditional behavioral theories are important to the development of persuasive technologies, a critical design component is often ignored. That critical, and perhaps unobvious, component is how technical support for behavior change impacts the individual's social world, as these technologies often must effectively integrate into the individual's everyday life. They are likely to be used in various circumstances, often crossing personal and work life. They inherently encroach upon the individual's social world and must therefore consider the social implications for the individual's daily experiences.

Thus, in this paper, we use concepts from behavioral and social psychological theories to shape an understanding of how to design technology to support behavior change while supporting fundamental social needs. We draw from Presentation of Self in Everyday Life [9] and Cognitive Dissonance Theory [6] in addition to the theories above. We propose design strategies for technologies that motivate behavior change in everyday life that specifically account for the intersection of the technology with the individual's social world. Our strategies extend four existing design goals through the application of the theories and findings from recent persuasive technology research. Our extensions account for characteristics that must be supported when a personal technology is used throughout everyday life. We used these strategies to design a system that encourages a physically active lifestyle. Two field studies of our system - a three-week trial and a three-month experimenthave shown that the system was successful at helping people maintain a more physically active lifestyle and validate the usefulness of our strategies on behavior change. 
We begin by recalling key aspects of the theories upon which our design strategies are based, focusing on the social psychological theories. We describe persuasive technology research that has influenced our strategies, and build on the discussion of theory and recent persuasive technology research by proposing our design strategies. We then present our system and discuss key results from our studies which validate that the proposed strategies are useful.

\section{OVERVIEWS OF THE THEORIES}

Designing persuasive technologies that target long-term discretionary use throughout everyday life is challenging, especially when those technologies attempt to help individuals change their everyday behaviors. Interestingly, this task often remains challenging even when the individual wants to change. Fortunately, much work in psychology focuses on how human behavior can be shaped and influenced, as well as people's basic social functioning.

We draw from Goal-Setting Theory [12] and the Transtheoretical Model of Behavior Change [16] - two theories that are often used in persuasive technology and health intervention research. Goal-Setting Theory [12] describes how individuals respond to different types of goals and thus which tend to motivate well. For example, the individual needs to have decided that the goal is important to her. It should be set by the individual or participatively with the help of an expert (rather than being assigned to her with no rationale). It should be easy to gauge her progress and know when she has met her goal, and it should be challenging, yet something that she believes she can realistically achieve. Feedback and incentives should be provided as progress is made and not limited solely to goal achievement.

The Transtheoretical Model [16] describes the stages through which an individual progresses to intentionally modify addictive or other problematic behaviors: Precontemplation-no intention to change in the foreseeable future; Contemplation-seriously considering changing, but has not committed to taking action; Preparation-intends to take action in the next month and has unsuccessfully taken action in the past year; Actionhas performed the desired behavior consistently for less than six months; and Maintenance-has consistently performed the desired behavior for six or more months.

The Transtheoretical Model suggests that a persuasive technology that targets precontemplators might focus on education. For contemplators, the design might focus on techniques for overcoming barriers or rewards for performing the desired behavior. For preparation stagers, it might focus on rewarding behaviors, even when the behavior is not consistent and increasing awareness of patterns of the behavior to encourage consistency. For action stagers, the design might focus on keeping track of progress to maintain consistency and possibly incorporate elements of social influence. For maintainers, it might focus on coping strategies for problems encountered previously and helping the individual realize how she is becoming "the kind of person one wanted to be" [16, p.12].

However, because individuals are likely to use the technology throughout everyday life, we turned to social psychology for theories that focus on how individuals manage their daily behaviors as part of a larger social context. Thus we adopt Presentation of Self in Everyday Life [9], which addresses the social interactions that individuals manage daily. And because the desire for lifestyle change often emerges when the individual recognizes a conflict between her current and an ideal state, we also use Cognitive Dissonance Theory [6], which describes what happens when an individual realizes that her behaviors and attitudes are inconsistent.

The application of Presentation of Self and Cognitive Dissonance Theory to persuasive technology is novel, however use of the theories in research and practice is not. For example, Aoki and Woodruff use Presentation of Self to analyze online community interactions and develop collaborative systems [1]. While the HCI community has yet to adopt Cognitive Dissonance Theory, some therapeutic approaches to lifestyle behavior change rely on it. In this paper, we illustrate how ideas from these two theories can be valuable for persuasive technologies.

In the remainder of this section, we recall useful concepts from Presentation of Self and Cognitive Dissonance Theory and implications of these theories to the design of technologies that support lifestyle behavior change.

\section{Presentation of Self in Everyday Life}

Presentation of Self in Everyday Life [9] describes how individuals attempt to manage the impressions they want others to have of them. This impression management is a constant process. Presentation of Self uses the metaphor of the theatre stage to describe how people interact with others. The performance encompasses "all the activity of an individual which occurs during a period marked by his continuous presence before a particular set of observers" [9, p.22]. The individual performs for an audience. The audience consists of those who observe the performance. The individual and audience are the participants in the performance. Non-participants are outsiders. The individual has a personal front, which consists of traits such as gender, age, size, looks, and clothing.

A given performance has two regions: front and backstage. Front stage is where the individual knowingly performs. Backstage is "a place, relative to a given performance, where the impression fostered by the performance is knowingly contradicted as a matter of course...Here the performer can relax; he can drop his front, forgo speaking his lines, and step out of character...[it is] where the performer can reliably expect that no member of the audience will intrude" [9, p.112]. Impression management describes how the individual moves between front and backstage and controls access to backstage. 
Other important concepts include dramatic realization, misrepresentation, and secret consumption. Dramatic realization occurs when the individual draws attention to facts that may go unnoticed. Misrepresentation suggests that individuals may be incented to misrepresent facts. Secret consumption explains that, "If an individual is to give expression to ideal standards during his performance, then he will have to forgo or conceal action which is inconsistent with these standards. When this inappropriate conduct is itself satisfying...then one commonly finds it indulged in secretly; in this way the performer is able to forgo his cake and eat it too" [9, p.41]. Secret consumption often results from idealization-the idealized impression the individual may attempt to convey of her behaviors.

\section{Implications of Presentation of Self}

Technology to encourage lifestyle behavior change must support fundamental impression management needs. Effective support implies that the individual should be in control of information about her that is collected and how that information is used. For example, it may be important to provide the ability for the individual to disguise something about her activities. The technology may also need to enable the individual to misrepresent something about her behavior, perhaps to support secret consumption.

It is often the case that a technology that seeks to represent "perfect information" may not effectively support an individual's basic need to control backstage access, yet it is important to give individuals control over their backstage. The technology needs to enable the individual to perform differently for different audiences. If the technology allows an audience member or outsider to access her backstage, the individual may perceive a violation of her privacy, which could result in her abandoning the technology.

\section{Cognitive Dissonance Theory}

Cognitive Dissonance Theory [6] explains what happens when an individual realizes that her attitudes and behaviors are inconsistent. When that happens, the individual will experience psychological discomfort (or dissonance). Because this dissonance is psychologically uncomfortable, the individual will be motivated to reduce or eliminate the dissonance. Her motivation depends on how important the beliefs or behaviors are to her. That is, the more important they are to the individual, the more likely she will try to reduce or eliminate the dissonance.

When an individual is motivated to reduce or eliminate dissonance, she can change her behavior or knowledge. She may also reduce the importance of the dissonance by actively learning about other things that are more harmful than whatever is causing the dissonance or by actively avoiding information or situations that may produce (or reinforce) the dissonance. For example, a smoker may experience cognitive dissonance because of the health risks she believes smoking causes. She can change her behavior-stop smoking, change her knowledge-stop believing that health risks are involved with smoking, rationalize the dissonance-focus on how she does not behave in ways that she can convince herself are more harmful than smoking, perhaps binge eating, or avoid information and situations that reinforce the dissonanceavoid warnings of smoking's health risks.

\section{Implications of Cognitive Dissonance Theory}

Cognitive Dissonance Theory suggests that a persuasive technology to encourage lifestyle behavior change should address whichever factors may prevent the individual from incorporating the change into her everyday life (i.e., by helping her change her behavior to match her attitudes). For example, the technology should help the individual remain focused on her commitment to change and her relevant patterns of behavior. The awareness provided by the technology should be persistently available and easy to access, yet subtle enough so as to support occasional needs for information/situation avoidance.

Next, we describe persuasive technology research that has also influenced the development of our design strategies.

\section{RECENT PERSUASIVE TECHNOLOGY RESEARCH}

Recent persuasive technology projects have attempted to help people achieve behavior change. In the following, we highlight several investigations that influenced the development of our proposed design strategies.

\section{Breakaway}

Breakaway [10] encourages the user to change behavior at work by getting up and taking a break. A sculpture that sits near the user's monitor slumps over as she continues to sit without taking a break. It provides peripheral awareness to the user when she is at her desk. If she takes regular breaks, the sculpture sits upright and appears "healthy." If she does not, the sculpture slowly slumps over. A pilot evaluation with a single participant (one week with and another without Breakaway) confirmed that the participant appreciated that she could ignore Breakaway during busy times-something her electronic calendar did not permit. She also took more breaks while using Breakaway.

\section{Fish'n'Steps}

Fish 'n'Steps [11] encourages users to take more steps each day. The user's daily step count (as measured by a pedometer) is linked to the emotional state, growth, and activity of a virtual fish displayed in a virtual fish tank with the fishes of other users on a kiosk in a common area of the user's workplace. Individual progress is accessible through a web site. Progress toward the user's step count goal affects the growth of her fish (the higher the step count, the larger the fish) and the fish's facial expression (happy for sufficient, angry for near-sufficient, and sad for insufficient progress). Insufficient progress from any user in the tank may result in murky water and the removal of decorations. A six-week deployment of the system $(n=19)$ that was compared to pre- and post-intervention baselines using pedometers only for eight additional weeks revealed that some participants ignored the display when their fish was not happy, causing the researchers to rethink the use of punishment. Results also revealed that several participants 
who wanted to increase their step count did so and the appealing design garnered interest from others.

\section{Houston}

Houston also encourages users to take more steps each day [3]. A small group of users share their step counts, as determined by a pedometer, and performance toward a daily step count goal via their mobile phones. They can add notes to their step counts, send messages, and review trending information. A congratulatory message and an '*', next to their step count reward each user for achieving her goal. A pilot evaluation $(n=13$, one week of baseline and two weeks with the full system) found that most participants increased awareness of their activity levels. They were motivated by simple rewards for achieving their goal (e.g., the'*'). Several explained that their step counts did not represent their activity levels, as pedometers do not detect activities such as bicycling and rock climbing, and they do not detect step count if the user is wearing a dress and therefore has no place to put it. The participants pointed out that receiving credit for all relevant activities is critical.

Next, we present our strategies for designing technologies to encourage lifestyle behavior change that were driven by the theories and research projects discussed above.

\section{DESIGN STRATEGIES FOR}

\section{LIFESTYLE BEHAVIOR CHANGE TECHNOLOGIES}

As we have argued, designing technology to help people get from the lifestyle they have to the lifestyle they want is complex. In the following, we present a set of strategies to inform the design of such technologies. We started with four design goals established by Breakaway [10]: abstract, non-intrusive, public, and aesthetic (Table 1). Breakaway’s original goals were specific to the Breakaway project-they were not presented as general design goals for technologies to encourage everyday behavior change. In many cases, our extensions to those goals involve generalizing them to apply to everyday behavior changes, we validated our proposed strategies in two field studies (discussed later), and we use the theories and research projects described above to explain the rationale for each strategy. In addition,

1. Abstract. We wanted to use data abstraction, rather than raw sensor data collected from the user, to display information to encourage people to be more active and to draw attention to the harm that is done to the body by sitting for long stretches of time;

2. Non-intrusive. We wanted to present data in a nonobtrusive manner and to make it available at all times during the work day without interrupting work;

3. Public. We wanted to present data in a way that people would willingly display it in a public place like the office. We needed to present the data, which is personal by nature, in a way that is appropriate to be presented in a public environment; and

4. Aesthetic. Since the display would function as a personal object within the office space, it would need to be inquisitive and sustaining interest over time.

Table 1. Breakaway's four original design goals [10], which we extended to develop our proposed strategies. we introduce four new strategies, which also incorporate concepts from the theories and research projects described above. Our eight proposed strategies are: 1) Abstract \& Reflective, 2) Unobtrusive, 3) Public, 4) Aesthetic, 5) Positive, 6) Controllable, 7) Trending / Historical, and 8) Comprehensive. We note that the strategies can overlap at times and are not meant to be mutually exclusive. We explain each strategy in turn.

1. Abstract \& Reflective. Use data abstraction, rather than raw or explicit data collected from the user and any technologies, to display information to encourage the user to reflect on his/her behaviors by showing the user what s/he has done and how those behaviors relate to his/her goal.

Abstracted data helps the individual with the task of impression management [9] by providing flexibility in the data's representation. This flexibility allows the individual to create alternative explanations of the data should it intentionally or otherwise be seen by another-that is, it provides ambiguity to create a "story" [1] —and it enables the individual to have the representation with her in various situations, thus increasing the opportunity for reflection.

Using abstraction to represent behavioral data to the individual was employed by Breakaway [10] with the sculpture that represented how long the user had been sitting without taking a break and Fish'n'Steps [11] with the fish tank display that represented the user's step count.

2. Unobtrusive. Present and collect data in an unobtrusive manner, and make it available when and where the user needs it, without unnecessarily interrupting his/her everyday life or calling attention to him/her.

To support impression management, the technology should not draw unwanted or unnecessary attention from or to the individual, despite being available when and where she needs it and in time to modify her behavior. Houston [3] addressed its goal of being unobtrusive by using a common technology that the individual already carries and uses in a wide variety of places-her mobile phone.

The technology should also support occasional ignorability as suggested by Cognitive Dissonance Theory [6] and evidence from Breakaway's pilot study. Breakaway allowed the study participant to ignore the technology when she was particularly busy (i.e., when something else temporarily took priority)—a feature that she specifically mentioned was important.

3. Public. Present and collect the data, which is personal in nature, such that the user is comfortable in the event that others may intentionally or otherwise become aware of it. Because the data needs to be available whenever and wherever the user needs it, it is likely to be something that s/he wears/carries, resides in a shared/common space, or uses while in the presence of others. The technology should not make the user uncomfortable in those situations. 
There is always a chance that a technology intended for personal use may be noticed by others, especially if it is being used throughout everyday life. This is particularly important when the data represents behaviors from front and backstage, and potentially across different audiences. Breakaway used data abstraction so that its sculpture could be in the presence of others without drawing attention to it or the individual. Because Houston runs on the individual's mobile phone, and individuals already use their mobile phones in a wide range of settings and are familiar with the social norms of using phones in public, it is unlikely that the individual would draw unwanted attention to herself while discretely interacting with the technology.

4. Aesthetic. If the display and any accompanying devices function as a personal object(s) that may be used over time, they need to be inquisitive and sustain interest. The physical and virtual aspects of the technology must be comfortable and attractive to support the user's personal style.

The "Aesthetic" strategy ties directly to the concept of personal front from Presentation of Self, as personal front includes the individual's appearance. If any aspect of the technology is worn or carried by the individual or displayed in her personal area, its aesthetics and comfort must reflect her personal style. Aesthetics can also help with the technology's surface credibility. Fogg notes that people often "assess the credibility of computing products based on a quick inspection of such surface traits” [7, p.133].

A success in Fish'n'Steps was the look of the fish when it was happy. Not only did a happy fish make participants feel good, but the aesthetics also drew interest and created excitement from others who were not participating but could see the kiosk display. Conversely, participants in the Fish'n'Steps and Houston studies complained about the ugliness of the pedometers that they wore.

5. Positive. Use positive reinforcement to encourage change. Reward the user for performing the desired behavior and attaining his/her goal. When the desired behavior is not performed, the user should not receive a reward nor a punishment, but his/her interest should be sustained.

A critical issue for technologies that encourage lifestyle behavior change is that they are likely to be long-term, discretionary use technologies - that is, the individual chooses when and if to use them. When actual behavior does not reflect desired behavior, the technology should not reward nor punish the individual, however it must sustain her interest. This is particularly important for technologies that are intended for use over long periods of time. Individuals occasionally get sick, injured, or have a deadline that legitimately prevents them from performing the desired behavior. Other times, they just need a break, perhaps to accommodate the basic need for secret consumption. At such times, the technology must sustain the individual's interest without making her feel too bad or it runs the very real risk of being abandoned.
Several existing behavior change technologies reward behavior. For example, Houston provides simple rewards upon goal attainment (e.g., an '*' and a congratulatory message). Similarly, Fish'n'Steps provides a happy fish, tank decorations, and clean water. Results from Houston's study showed that individuals appreciated the rewards and found them to be surprisingly motivating. However, the use of punishment in some projects—-such as Fish'n’Steps' sad or angry fish, murky water, and removal of tank decorations—-sometimes resulted in reduced use.

6. Controllable. When appropriate, permit the user to add to, edit, delete, and otherwise manipulate data so that it reflects the behaviors that s/he deems suitable. The user should be in control of who has access to what aspects of his/her data

Whether the information is manually entered by the individual, automatically detected by a monitoring technology, or some combination of the two, the system should permit the individual to manipulate the data and give her control over who has access to what data. This can help overcome limitations of technologies that automatically infer behavior and gives the individual control over access to her backstage. Further, allowing the individual to manipulate her data facilitates dramatic realization, misrepresentation, and secret consumption. A tricky problem is how to accommodate such concepts while helping the individual reflect on her actual behaviors.

The issue of the limitations of technologies that automatically infer behavior has two important components: (1) individuals will be upset when a technology does not give them proper credit for behaviors they do that are in support of their desired lifestyle, and (2) individuals may be frustrated when they receive credit for something they did not do. Although the problem of getting credit for something one did not do is less obvious than the problem of not getting credit for something one did do, it is nevertheless important. For example, if the system reflects that the individual is burning enough calories each day to lose two pounds per week when she is only burning enough to maintain her weight, she may have difficulty realizing why she is not experiencing the expected weight loss and get frustrated. Fogg warns that the issue of inaccurate data representation may lead to a loss of credibility [7, p.127]:

Credibility is key when computing products report measurements. If reported measurements are questionable or obviously inaccurate, the products will lose credibility. If the product were designed to influence or motivate, it likely would fail because of the inaccurate measurements it had reported.

Participants in Houston's study complained about missed steps in their daily step counts when they forgot to wear their pedometers or when they were wearing a dress and had no reasonable place to clip the pedometer. This shows that sometimes the usage model of the technology, and not its accuracy, causes problems. 
7. Trending / Historical. Provide reasonable and accessible information about the user's past behavior as it relates to his/her goals. Historical data should accommodate changes in lifestyle goals over time and provide for the portability of data across devices.

The seemingly mundane choices that individuals make every day often impact whether they achieve their desired lifestyle. As aforementioned, this is complicated by the fact that infrequently making a poor or arbitrary decision is seldom a disaster. Rather it is a pattern of such decisions that typically prevents the achievement of lifestyle goals [17]. If the individual considers each decision in isolation, and not based on a history of decisions, it is easy for her to make a "poor decision," as it often leads to immediate satisfaction. This point is echoed by Cognitive Dissonance Theory's concepts of information/situation avoidance and magnitude, as the individual may try to reduce dissonance by deliberately ignoring the impact of the pattern of her behaviors. Technologies to encourage lifestyle behavior change should help the individual reflect on her past behaviors in relation to her goal. This historical perspective will help the individual "keep her eyes on the prize."

Houston provided short-term trending information, which was used and appreciated by the participants.

8. Comprehensive. Account for the range of behaviors that contribute to the user's desired lifestyle; do not artificially limit data collection and representation to the specific behaviors that the technology can sense or monitor.

With Fish'n’Steps and Houston, pedometers were used to measure behavior and subsequently calculate goal progress. If the individual went for a bike ride, a desirable behavior regarding a "physically active" lifestyle goal, the individual would not receive credit toward her goal. Participants in Houston's study specifically complained about that situation, and explained that it discouraged them from performing some healthy behaviors. For example, why go for a run when walking will yield a higher step count for less effort? Why go for a bike ride when those activities will yield no step count? Houston's results point to an important side effect of the system not properly accounting for relevant behaviors. That is, Houston was trying to help individuals focus on being active, but for several participants, the focus turned to optimizing their step count. This change in focus often resulted in participants doing less healthy activities than they had planned.

This point is particularly important for individuals in the contemplation, preparation, and action stages of the Transtheoretical Model who are attempting to establish routines. Discouraging an individual in one of these stages from performing a relevant behavior may inhibit progress and result in her reverting back to a prior stage.

A note about collaborative technologies. In this paper, we focus on supporting an individual. Technologies to support lifestyle behavior change may incorporate collaborative elements, such as features provided by Fish'n'Steps and Houston. While our theoretical basis and strategies do not preclude collaboration, we caution that moving from designing for an individual interacting with a technology to designing for collaboration (even if the focus remains the individual) requires careful consideration.

\section{IMPLEMENTING THE DESIGN STRATEGIES: THE UBIFIT GARDEN SYSTEM}

In this section, we describe a system that we developed using our proposed strategies. During our initial design phase, we engaged the aforementioned theories, developed the design strategies, then used the strategies to inform the design of our system. Our strategies were refined as we conducted field studies of the system (discussed later). The persuasive technology we present here, the UbiFit Garden system, encourages individuals to lead a physically active lifestyle. We believe that the design could be modified to support other lifestyle behavior changes.

UbiFit Garden uses the screen background, sometimes called “wallpaper," of an individual's mobile phone to display a garden that blooms as she performs physical activities throughout the week. Upon meeting her weekly goal, a butterfly appears. Smaller butterflies represent goals attained from the past three weeks. An interactive application on her phone includes detailed information about the individual's physical activities and a journal where she can manually add, edit, and delete information about her activities. A fitness device (we used the Mobile Sensing Platform (MSP) [2]) automatically infers and communicates information about walking, running, cycling, using the elliptical trainer, and using the stair machine to the garden display and interactive application (Figure 1). More details can be found elsewhere [4,5].

UbiFit Garden implements the design strategies as follows:

1. Abstract \& Reflective. UbiFit Garden's display uses an animated garden as a metaphor to represent physical activity behavior and goal attainment. The data abstraction - the garden-being on the background screen of the individual's mobile phone affords frequent reflection that supports the individual's desire to live a physically active lifestyle. At a glance, she can determine if she is
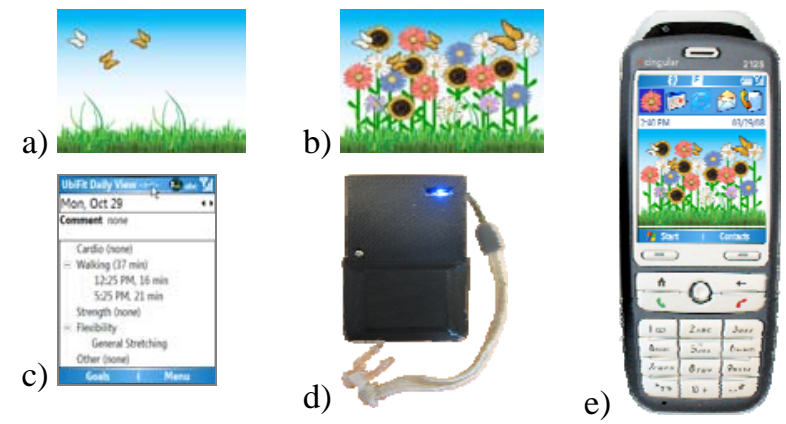

Figure 1. The UbiFit Garden system. a) The garden at the beginning of the week; b) an active week with variety; c) the interactive application; d) the MSP; and e) the garden on the background screen of a mobile phone. 
having an active or inactive week (based on the number of flowers), if she has incorporated variety into her routine (the variety of flowers), if she has met her weekly goal (the presence of a large butterfly), and if she has met her goal recently (the presence of one or more small butterflies).

2. Unobtrusive. The garden display resides on the background screen of the individual's mobile phone where it conveys key information about her behavior and goal attainment. The interactive application also resides on the individual's mobile phone. Due to their popularity, mobile phones are essentially available whenever and wherever the individual is. Because individuals already use their phones often, they will see the background screen often. Individuals will not stand out as different for using a mobile phone, nor will they have to take new or additional action to see the garden display ${ }^{1}$.

3. Public. Because mobile phones are frequently used in social situations, their screens may intentionally or unintentionally be seen by others. The abstract nature of the garden display supports the public strategy. For example, if someone unintentionally sees the individual's background screen, she could describe the image in any number of ways, including it simply being a picture.

4. Aesthetic. People frequently change the default background screen of their phones to an aesthetic image or photograph. Because the garden display uses a feature that is traditionally aesthetic only and replaces it with something that has utility, its design must not only be functional, but also aesthetic. Therefore, the garden display has been designed to look like something that an individual might use as her personal background screen.

5. Positive. UbiFit Garden uses rewards to encourage behavior. When the individual performs an activity, a flower blooms in her garden. If she meets her weekly goal, a large butterfly appears. Smaller butterflies indicate when goals were met for the prior three weeks, therefore the individual can see up to a month's worth of goal attainments in her garden. If she performs activities but does not reach her goal, she simply does not get a butterfly. If she does no activity whatsoever, she will have an empty garden with green grass and a blue sky. The flowers do not die. The grass does not wilt. The sky does not storm. The individual also gets a fresh start each week when the screen resets to an empty garden, so even if she had a bad week, she can start over fresh in the coming week.

6. Controllable. UbiFit Garden's interactive application allows the individual to add, edit, or delete any information about her recorded activities, whether those activities were manually entered by her or automatically inferred by the

\footnotetext{
${ }^{1}$ The MSP is an early-stage research prototype, and thus is larger than its envisioned form factor. As such, it did not meet the criteria of being Unobtrusive, Public, or Aesthetic. These complications are typical of early-stage research prototypes.
}

fitness device. Through this application, the individual can also see traditional text and numerical descriptions of her activities and add comments to her daily list, for example, “CHI deadline tomorrow!"

7. Trending \& Historical. The garden display represents a week's worth of activities to allow the individual to reflect on her performance over the space of a week. The small butterflies indicate goal attainment for recent weeks, serving to reward and remind her of up to a month's worth of successes. The interactive application also allows individuals to reflect on a longer view of past performance.

One idea that was not implemented in the UbiFit Garden prototype is that the display and interactive application could be mirrored on a traditional desktop software application or on a web site. Additional features that are less suited to the phone's small screen and limited text entry capabilities could be added to a more traditional software/web site component. For example, longer term trending charts and images could be useful for individuals as they use the technology over longer periods of time.

8. Comprehensive. The MSP has been trained to infer several types of commonly performed physical activitieswalking, running, cycling, elliptical trainer, and stair machine. Most current fitness devices do not detect such a range but are limited to measurements such as step count (e.g., pedometers), running \& walking durations/distances (e.g., Nike+ iPod and the Nokia 5500 Sport), or cycling durations/distances (e.g., bike computers). However, because the MSP does not detect the full range of activities that are relevant to a physically active lifestyle, the interactive application allows individuals to manually enter any physical activity of their choosing, and they have flexibility over how those activities are categorized (e.g., if the individual believes that mowing her lawn qualifies as "cardio" and not "other," she may journal it as such).

\section{VALIDATING THE STRATEGIES WITH UBIFIT GARDEN}

We have used an iterative design process to develop the UbiFit Garden system and validate our design strategies. This process included a paper-based survey, a 3-week field trial, and a 3-month field experiment. The survey included a mix of multiple choice and open-ended questions about respondents' use of mobile phones, their physical activity goals and practices, and two proposed designs. Seventy-five people (46 female) who ranged from 18 to 63 years old and lived in 13 states across the U.S. responded.

In the three-week field trial, 12 participants (six female) who were recruited from the general public used the UbiFit Garden system for 21 to 25 days. Participants were from 25 to 35 years old, lived in the Seattle Metropolitan area, and were regular mobile phone users who wanted to increase their physical activity. In the three-month field experiment, 28 participants (15 female) who were recruited from the general public used one of three versions of the UbiFit Garden system for three months over the winter holiday season. Participants were aged 25 to 54, lived in the Seattle 
Metropolitan area, and were regular mobile phone users who wanted to increase their physical activity. Three experimental conditions (Full System, No Garden, and No MSP) helped us determine the impact of the garden display and MSP. Additional details can be found in [4,5].

Reactions to UbiFit Garden have been overwhelmingly positive. Everyone who used the garden display thought that it (or a different metaphor) was essential, and most who had not used it wished they had when they learned about it at the end of the study. The garden metaphor was an understandable representation of physical activity behavior and goal attainment. In fact, several participants explained how even small children understood it.

The garden's effectiveness was further supported by a statistically significant analysis that showed that participants in the Full System and No MSP conditions (i.e., versions of the system with the garden) maintained their weekly activity duration on average throughout the three months, even on weeks that contained a holiday, while the activity duration for those who did not have the garden went down over time and on holiday weeks.

For more detail, including a discussion of the statistically significant findings, please see [4].

\section{Evidence Linking UbiFit's Design to Design Strategies}

In this section, we highlight key results from the studies that address our design strategies. For reasons of space, we focus on findings from the three-month field experiment and often use representative quotes to describe the participants' perspectives in their own words.

1. Abstract \& Reflective. Participants who used the garden display commented on how they appreciated that unless they chose to reveal what their garden meant, someone who saw their phone would not know. Participants also explained how because the garden was on the background screen of their phone, they thought about their physical activities and commitment to being active more than usual. One participant observed how it reminded her to be active:

[The garden] was a constant reminder...whereas if you didn't have a [garden], you probably_l wouldn't think about [physical activity] as much, you know. [With the garden] I think about it maybe subconsciously every time I look at my phone.

2. Unobtrusive \& 3. Public. All participants agreed that the mobile phone was a desirable place for the garden display and interactive application, as it integrated well with their everyday lives. As one participant explained:

I liked having [my garden] be on the phone...something I have with me...[With] a Web site, it's so easy, 'oh, I didn't do anything, I'm not going to click on it.' It's so easy to ignore it. But on the phone, you can't really ignore it as easily.

Additionally, no participant left his/her phone behind or refrained from using it in public because of UbiFit Garden.
However, due to the early nature of the MSP prototype that we used, it often did not meet the criteria for being Unobtrusive or Public. Most participants who used the MSP recounted anecdotes of being asked about it. Although they were often not uncomfortable by the inquiries per se, it got tiresome over time. Participants established practices where if they were concerned that the MSP would be distracting (e.g., when presenting in a meeting or teaching a class), they would take it off or leave it behind.

4. Aesthetic. Many of the participants found the garden display to be attractive and motivating:

[ 1 liked]...the little rewards [the flowers and butterflies]... When [the garden] starts filling up with flowers, [it's] very nice.

Receiving the pretty flowers and butterflies for being active made participants feel good about what they had accomplished. In fact, participants' children liked the garden too, which often served as an indirect motivator:

[My daughter] would really encourage me to [be active] and she would ask me for pink flowers all the time... She was very excited, and she wanted [me to get] the butterflies.

However, though most participants wanted a display that worked like the garden, the garden itself did not always accommodate personal styles, particularly for the men. For example, some of their friends teased them about having flowers on their phones. One male participant explained:

[I was] hanging out with a couple of buddies that I haven't seen for a while, they're like, 'Oh, nice garden there.'

Participants occasionally thought that the MSP clashed enough with their outfits that they did not wear it (e.g., when going out to dinner and drinks or to church), illustrating that when personal style is not supported by the technology, the technology will often be left behind.

5. Positive. Many participants commented on the positive nature of the flowers, and how they appreciated that it was a gentle reminder to be active. As one participant explained:

It's a nice, gentle reminder that getting off my duff... every other day is not too much to ask.

As mentioned earlier, for behaviors such as physical activity, individuals are unlikely to perform the desired behavior all of the time for any number of reasons, many of which are perfectly legitimate ${ }^{2}$. Participants encountered barriers throughout the field studies - several of which were out of their control-that led to their not performing as much physical activity as they had hoped. One participant described what it was like to be sick:

\footnotetext{
2 Taking an occasional break is often considered to be legitimate, and in fact often helps with being able to sustain the behavior over time, as long as the break does not become a pattern [17].
} 
It was awful...I was like counting the days since I had been to the gym or since I had done anything because I had some really sick days where just stretching made me want to throw up.

Individuals already feel bad when they do not meet their goals; they do not need the persuasive technology that is trying to help them instead make them feel worse. Most participants in our studies were able to get back on track, which emphasizes the value of trying to sustain their interest and gently remind them of the commitment they made without punishing them for the lapse in behavior.

6. Controllable. In the two field studies, participants actively journaled activities, and edited and/or deleted data about inferred activities. All participants who used the MSP thought that those features were essential. In many cases, they made inference errors tolerable, as participants were able to correct mistakes, whether it was an error of confusion, omission, or credit for something unearned. They also permitted participants to receive credit for activities performed when the MSP was not charged or had been left behind. Participants stressed the importance of their activity record being accurate, as an inaccurate record would only hurt them. However, despite the occasional problems with the activity inference, most found it useful.

7. Trending / Historical. We only have limited evidence to support Trending / Historical, given that three months is not very long when considering a lifetime. However, participants commented on how the month's worth of goal attainments motivated them:

I could see my progress, if I was-how much more I needed to do to get to my goal...And I could see the butterfly and think, "I did it last week, you can do it again this time.'

Another participant explained how the week's worth of activities shown in the garden kept her honest about what she had done and still had to do:

I used [the garden] to increase my awareness of what I was doing...'cause like... after about two days, you kind of forget, like 'did I really do that or am I just dreaming, or was that last week?'

Although a longer-term study, probably with an accompanying web site to accommodate limitations of viewing charts and other effective representations of trending information on the mobile phone, is needed to provide stronger evidence to support the Trending / Historical strategy, results from the field studies confirm that at least short-term trending is useful.

8. Comprehensive. Participants performed 532 activities in the three-week field trial and 1853 activities in the threemonth field experiment, $35-40 \%$ of which were automatically inferred by the MSP and the remainder of which were manually journaled by the participants. In both studies, participants recorded a broad range of physical activities. In the three-month field experiment, 26 types of cardio activities alone were recorded. Unlike in the Houston study [3], no participant in the UbiFit Garden studies refrained from doing an activity because of the system. These findings suggest that when the technology accounts for the range of relevant behaviors, individuals do not artificially limit themselves to the behaviors that the technology can automatically sense.

\section{RELATED WORK}

Above, we mentioned specific projects that directly influenced our proposed design strategies. In this section, we describe other related efforts that use technology to encourage healthy lifestyle behavior change.

Similar to Houston, two other projects that use mobile phones to facilitate the exchange of physical activity measurements amongst small groups of users are Chick Clique [18] and Shakra [13]. Chick Clique uses mobile phones and pedometers to help teenage girls share their step counts. Shakra uses mobile phones to help adults share their daily physical activity durations as well as to determine estimates of those durations based on the mobile phone's travels. Similar to Houston, Shakra experienced credibility issues with automatic activity measurements during a oneweek pilot study. Shakra's sensing technology sometimes mistook healthy behavior for sedentary behavior, thus resulting in inaccurate representations of behavior that could not be corrected in the system by the participants.

ViTo [15] replaces the television (TV) remote control with a hand-held device to help the individual decrease TV viewing time and increase physical activity. In addition to channel navigation, ViTo offers alternatives to watching TV, including listening to music, viewing a "to do" list, and playing isometric conditioning games. The researchers emphasized, "elements of fun, reward, and novelty are used to induce positive affect rather than feelings of guilt" [15, p.301], which is consistent with our Positive strategy.

Gasser et al.'s lifestyle coach [8] provides users with feedback about their physical activities and dietary intake. A 28-day field study $(\mathrm{N}=40)$ compared the persuasiveness of the mobile application to an equivalent web application. Results revealed no significant differences across conditions. However, the analysis did reveal a strong relationship between pre-existing motivation for lifestyle behavior change and goal achievement (similar to Fish'n'Steps). Results also revealed that the mobile application was used throughout the day, more effectively incorporating with everyday life than the web applicationwhich supports our Unobtrusive strategy.

\section{CONCLUSION}

We are exploring how to design technology to help people transition from the lifestyle they have to the lifestyle they want by helping them change their behavior. In this paper, we made two primary contributions. First, we proposed a set of design strategies for technologies that motivate lifestyle behavior change by using theory and findings from recent persuasive technology research to substantially 
extend a set of existing design goals. We employed two theories that are not traditionally used in the development of persuasive technologies-Presentation of Self in Everyday Life [9] and Cognitive Dissonance Theory [6]. Second, we described how our UbiFit Garden system, which motivates a physically active lifestyle, was designed based on the strategies. We highlighted results from our studies, focusing on results from our three-month field experiment, which confirmed that the system was successful at helping people maintain physical activity and validated the usefulness of the design strategies.

It is important for technology designers to recognize that lifestyle behavior change is a long-term endeavor that pervades everyday life, including the social world. If done poorly, the technology is likely to be abandoned; therefore a principled approach for its design is needed. This paper presents such an approach based on theory and prior results that we are using in a larger research agenda to design persuasive technologies to affect lifestyle behavior change.

\section{ACKNOWLEDGEMENTS}

We would like to thank our many colleagues who helped develop and support the UbiFit Garden system, particularly Beverly Harrison, Pedja Klasnja, and Tammy Toscos who were instrumental in running the field studies. We would also like to thank the study sponsor, Intel Research Seattle, the participants in the UbiFit studies, and the reviewers for their feedback.

\section{REFERENCES}

1. Aoki, P.M. \& Woodruff, A. "Making Space for Stories: Ambiguity in the Design of Personal Communication Systems.” Proceedings of CHI 2005, Portland, OR, USA, (Apr 2005), 181-90.

2. Choudhury, T., Borriello, G., Consolvo, S., Haehnel, D., Harrison, B., Hemingway, B., Hightower, J., Klasnja, P., Koscher, K., LaMarca, A., Landay, J.A., LeGrand, L., Lester, J., Rahimi, A., Rea, A., \& Wyatt, D. "The Mobile Sensing Platform: An Embedded Activity Recognition System," IEEE Pervasive Computing, 7(2), (Apr-Jun 2008), 32-41.

3. Consolvo, S., Everitt, K., Smith, I., Landay, J.A. "Design Requirements for Technologies that Encourage Physical Activity,” Proceedings of CHI 2006, Montreal, Canada, (Apr 2006), 457-66.

4. Consolvo, S., Klasnja, P., McDonald, D.W., Avrahami, D., Froehlich, J., LeGrand, L., Libby, R., Mosher, K., \& Landay, J.A. "Flowers or a Robot Army? Encouraging Awareness \& Activity with Personal, Mobile Displays," Proceedings of UbiComp 2008, (Seoul, Korea), (2008), 54-63.

5. Consolvo, S., McDonald, D.W., Toscos, T., Chen, M.Y., Froehlich, J., Harrison, B., Klasnja, P., LaMarca, A., LeGrand, L., Libby, R., Smith, I., \& Landay, J.A.
"Activity Sensing in the Wild: A Field Trial of UbiFit Garden,” Proceedings of CHI 2008, (Florence, Italy), (2008), 1797-806.

6. Festinger, L. A Theory of Cognitive Dissonance. Stanford, CA: Stanford University Press, (1957).

7. Fogg, B.J. Persuasive Technology: Using Computers to Change What We Think and Do, San Francisco, CA, USA: Morgan Kaufmann Publishers, (2003).

8. Gasser, R., Brodbeck, D., Degen, M., Luthiger, J., Wyss, R., Reichlin, S. "Persuasiveness of a Mobile Lifestyle Coaching Application Using Social Facilitation," Proceedings of Persuasive 2006, Eindhoven, The Netherlands, (May 2006), 27 - 38.

9. Goffman, E. The Presentation of Self in Everyday Life. New York, NY, USA: Doubleday Anchor, (1959).

10. Jafarinaimi, N., Forlizzi, J., Hurst, A., Zimmerman, J. "Breakaway: an ambient display designed to change human behavior," In CHI '05 Extended Abstracts, Portland, OR, USA, (Apr 2005), 1945-8.

11. Lin, J.J., Mamykina, L., Lindtner, S., Delajoux, G., Strub, H.B. "Fish'n'Steps: Encouraging Physical Activity with an Interactive Computer Game," Proceedings of UbiComp'06, Orange County, CA, USA, (Sep 2006), 261-78.

12. Locke, E.A. \& Latham, G.P. "Building a Practically Useful Theory of Goal Setting and Task Motivation: A 35-Year Odyssey,” Amer Psych, 57(9), (2002), 705-17.

13. Maitland, J., Sherwood, S., Barkhuus, L., Anderson, I., Hall, M., Brown, B., Chalmers, M., \& Muller, H., "Increasing the Awareness of Daily Activity Levels with Pervasive Computing," Proceedings of Pervasive Health '06, Innsbruck, Austria, (Nov/Dec 2006).

14. Mamykina, L., Mynatt, E., Davidson, P., \& Greenblatt, D. "MAHI: Investigation of Social Scaffolding for Reflective Thinking in Diabetes Management," Proc of CHI '08, Florence, Italy, (2008), 477-86.

15. Nawyn, J., Intille, S.S., \& Larson, K. “Embedding Behavior Modification Strategies into a Consumer Electronic Device: A Case Study," Proceedings of UbiComp'06, (Sep 2006), 297-314.

16. Prochaska, J.O., DiClemente, C.C., Norcross, J.C. "In search of how people change: Applications to addictive behaviors,” Amer Psych, 47(9), (Sep 1992), 1102-14.

17. Rachlin, H. The Science of Self Control. Cambridge, MA, USA: Harvard University Press, (2004).

18. Toscos, T. \& Connelly, K., "Encouraging Physical Activity in Teens: can technology help reduce barriers to physical activity in adolescent girls?,” Proceedings of Pervasive Health '08, Tampere, Finland, (2008). 